\title{
FRÜHBYZANTINISCHE BRONZEFUNDE AUS DEM THEATER VON NYSA AM MÄANDER
}

\author{
Özet \\ Nysa Tiyatrosu Erken Bizans Dönemi Bronz Buluntuları
}

\begin{abstract}
Nysa (Sultanhisar) tiyatrosunda sürdürülen kazı çalışmalarında 2000 yılında gün 1şığına çıkartılan bir grup bronz eser, bu makelenin konusunu oluşurmaktadır. 4. Podyumun temel blokları arasındaki bir boşluk içerisinde bulunan bu eserler, yapının kullanım sürecini belirlemek açısından önemlidir. Mimari bezeme, yazıtlar ve de Erken Bizans Dönemi yapılaşmasıyla belgelenen yapı evreleri, bu bronz eserlerle de desteklenmektedir. İlk evresi, Geç Hellenistik-Augustus dönemine tarihlenen Nysa tiyatrosu, sahne binası scaneae fronsu ile birlikte M.S. 2.yy'da (2. evre Hadrian döneminde [M.S.120-140] ve 3. evre Geç Antonin-Erken Severuslar döneminde [M.S.180-200]) iki kez yenilenmiştir. 2003 yılı kazılarıyla tamamen ortaya çıkarılan koridor ve hyposkenion tonozları, podyum temellerinin 3. podyum hariç aynı tür yapı malzemesi ile inşa edilmiş olduğunu göstermiştir. 3. podyum temelinde kullanılan kireçtaşından yapılmış, üç faskialı iki arşitrav bloğu, hem malzemesi hem de işlemesiz olmasından dolayı M.S.2.yy.'a tarihlenen arşitravlardan ayrılmaktadır. Bu, erken dönem malzemesinin 2. yüzyılda tekrar kullanılmış olduğunu gösterir. Böylece Augustus Dönemine tarihlenen iki adet bronz sikke, podyum temellerinin tarihlenmesi için kullanılamamaktadır. Bu durum, sikkelerin ya uzun süre kullanımda kaldıklarını ya da Erken Bizans Dönemi’nde eritilmek üzere toplanmış olduklarını düşündürmektedir. Buluntular arasında yer alan fibulanın ve Sirakuza tipli kemer tokasının, İmparatorluğun doğusunda da ortaya çıkması, dağılım açısından önem taşımaktadır.
\end{abstract}

Die karische Stadt Nysa am Mäander liegt am Abhang der Messogisberge, der heutigen Aydın Dağları, auf der Nordseite der Mäanderebene. Nahe der antiken Stadt befindet sich heute die im 13. Jh. entstandene Stadt Sultanhisar ${ }^{1}$.

Die von Sultanhisar kommende Zufahrtstrasse zur Ruinenstätte endet unmittelbar vor dem antiken Theater der Stadt, das heute noch ca. 10.000 Zuschauern Platz bietet ${ }^{2}$. Die Gesamtanlage, deren Grundmaße 72,6 x $98,8 \mathrm{~m}$ betragen, besteht aus dem sich an

1 Zur Geschichte der Stadt im Osmanischen Reich vgl. Wittek 1934; ders., Menteşe Beyliği ${ }^{3}$ (türkische Übersetzung von O.Ş. Gökyay) [1999] 22ff.; Akın 1968, 95ff.

2 Im Theater findet jedes Jahr das Nysa-Kulturfest statt. den nördlichen Berghang anlehnenden Zuschauerraum (cavea), der Orchestra, dem Proskenion und dem Bühnenhaus mit dreigeschossiger scaenae frons, das die Anlage nach Süden abschließt.

Die ersten Grabungen im Theater wurden in den 1980er Jahren durch das Archäologische Museum von Aydın durchgeführt und nur in kurzen Grabungsberichten veröffentlicht ${ }^{3}$. Seit Anfang der 90er Jahre unterstehen die Arbeiten in Nysa dem Archäologischen Institut der Universität Ankara unter der Leitung von V. İdil. Im Rahmen einer Dissertation über die Theaterfassade wurden die Arbeiten im Theater seit 1998 wieder

\footnotetext{
3 Sezer - Tuna - Peker 1988, 85-100; ders.1989, 307 322.
} 
aufgenommen ${ }^{4}$. Schwerpunkte der seit 1999 vorgenommenen Grabungen im Theater waren die Feststellung der Bauphasen und die Rekonstruktion der Schaufassade (scaenae frons). Das Theater von Nysa gehörte höchstwahrscheinlich zur Grundausstattung der hellenistischen Stadt und wurde in der römischen Kaiserzeit mehrmals umgebaut.

Die stilistische Analyse des Baudekors belegt drei Bauphasen des Theaters ${ }^{5}$. Nach der Profilabfolge, der Ornamentik der Bauteile und den Inschriften fällt die ältere Phase in die späthellenistisch-frühaugusteische Zeit ${ }^{6}$. Zwei weitere Bauphasen, die nur mit der scaenae frons und der Proskenionfassade in Verbindung zu bringen sind, folgten im 2. Jh. n. Chr. Die damals wohl noch zweistöckige Fassade wurde im Rahmen der zweiten Bauphase nach Aussage der ornamentierten Bauteile in hadrianischer Zeit erneuert ${ }^{7}$. Die dritte Bauphase ist durch den stilistischen Vergleich der Bauornamentik in spätantoninisch-frühseverischer Zeit (180 bis 200 n. Chr.) anzusetzen ${ }^{8}$.

Während der Grabungen im Hyposkenion (Schnitt H1) wurde im Jahr 2000 unterhalb der Türschwelle der porta regia zwischen den letzten Fundamentquadern des Podiums IV eine Gruppe von Bronzen ${ }^{9}$, bestehend aus sieben Münzen (Abb.1-7), einem Deckel (Abb. 1), dem Lichtteller eines Lampenständers (Zeichn.1, Abb. $8^{10}$ ), einer

4 Kadığlu 2002. Für die Überlassung des Materials zur Publikation schulden wir dem Grabungsleiter Dank. Zu den Grabungen vgl. İdil 2002, 77ff.

5 Kadığlu 2002, 151 ff. 247.

6 Kadıŏglu 2002, 154ff.

Kadıŏlu 2002, $165 \mathrm{ff}$.

Kadığlu 2002, 199ff.

9 Die Bronzen wurden von der Restauratorin A.E. Gültekin an der Başkent-Fachhochschule für Konservierung und Restaurierung der Universität Ankara konserviert.

${ }^{10}$ Hiermit möchten wir N. Kadıŏglu danken, die die Zeichnungen angefertigt hat.
Gürtelschnalle (Zeichn.2, Abb.9), einer Fibel (Zeichn.3, Abb.10), einer Verkleidungsklammer (Zeichn.4, Abb.11) und einem punzverzierten, pilzförmigen Bronzeblech (Zeichn.5, Abb.12) gefunden. Sie befanden sich in einem kleinen Hohlraum im Fundament auf Höhe der spätantiken Zumauerung der Öffnung unterhalb der porta regia, die den Durchgang vom Korridor zum Kellergeschoss ermöglichte. Die Füllung des Hohlraumes war einheitlich und ist als abgeschlossene, klar abgrenzbare stratigraphische Einheit $\mathrm{zu}$ bezeichnen. Daher sind die Funde wohl in frühbyzantinischer Zeit als Bronzeschrott eingesammelt worden, um sie anschließend einzuschmelzen und das Rohmaterial wiederzuverwenden. Aus einem heute nicht mehr nachvollziehbaren Grund wurden sie dann gemeinsam in dem Hohlraum niedergelegt und später nicht mehr geborgen. Nicht ganz auszuschließen, aber dennoch unwahrscheinlich ist, dass die aus verschiedenen Zeiten stammenden Funde innerhalb einer längeren Zeitspanne in den kleinen Hohlraum gerutscht sind und dort im eingeschwemmten Erdmaterial abgelagert wurden. Die Tatsache, dass die meisten Funde schon beschädigt und funktionsunfähig in die Erde gelangt sind, spricht aber ebenfalls gegen den zufälligen Verlust der Fundstücke und für eine gemeinsame Sammlung und Deponierung.

Durch die im Theater gefundenen Inschriften konnte bisher schon eine Nutzung des Baus bis in das 5./6. Jh. nachgewiesen werden, mit der geringe frühbyzantinische Bauaktivitäten korrespondieren ${ }^{11}$. Mit den Bronzefunden aus dem Hyposkenion kann nun auch eine Begehung des Gebäudes im 7. Jh. konstatiert werden, obwohl die Gewölbe des Hyposkenions, der Korridor zwischen den Fundamenten der Podien und den Hyposkenion-Gewölben und das Kellergeschoss des Bühnenhauses zu diesem

${ }^{11}$ Kadıoğlu 2002, 251ff. 
Zeitpunkt von heruntergestürzten Blöcken der Fassade und anderem Schutt schon fast vollkommen verschüttet waren.

\section{Fundkatalog $^{12}$}

\section{Inv.-Nr. TR-00 / 8a}

Pergamon, AE, (vor 133 v. Chr.).

Av Asklepioskopf im Lorbeerkranz nach $\mathrm{r}$. Rv Schlangenstab; A $\Sigma$ K $\Lambda$ HПIOY $\Sigma \Omega$ THPO $\Sigma$

Maße: Dm 1,6 cm, Stärke 0,3 cm, W 3,9 g Guter Erhaltungszustand. Vermutlich war sie in einen Deckel eingesetzt. Dunkelgrüne Patina.

Bibliographie: SNG v. Aulock 1957, Nr.1373 Taf.42; BMC Greek Coins 128, 150ff.; Voegtli, 1993, 28f. Nr.76. 81. 84 Taf.1.

\section{Inv.-Nr. TR-00 / 8b}

Deckel aus Bronze

Maße: $\mathrm{Dm} 1,8 \mathrm{~cm}, \mathrm{H}$ 0,8 cm

Gut erhaltener, runder Deckel. Auf der Innenseite noch Reste einer Eisenklammer und von Blei. Dunkelgrüne Patina.

Datierung: Frühbyzantinisch ?

\section{Inv.-Nr. TR-00/5}

Augustus, Provinz Asia, Dupondius, AE, (27-23 v. Chr.)

Av Augustuskopf nach r.

Rv CA im Lorbeerkranz .

Maße: Dm 2,7 cm, Stärke 0,3 cm, W 10,5 g. Pergamenische Prägung? Dunkelgrüne Patina. Schlechter Erhaltungszustand. In Folge der langen Benutzung sind die beiden Seiten stark abgenutzt. Auf der Rückseite ist nur noch der Lorbeerkranz schwer zu erkennen.

Bibliographie: RPC I 2230. 2234. Taf. 99; Arslan 1992, Nr.15.

\section{Inv.-Nr. TR-00/7}

Provinz Asia, Dupondius, AE, Augustus (27-23 v. Chr.)

Av Augustuskopf nach r.

Rv CA im Lorbeerkranz (unbestimmbar).

Maße: Dm 2,6 cm, Stärke 0,2 cm, W 7,6 g

Pergamenische Prägung? Vorder- und Rückseite korrodiert. Grüne Patina. Schlechter Erhaltungszustand. In Folge der langen

${ }^{12}$ Für die Hinweise zu den Münzen danken wir S. Doruk, K. Konuk, Th. Ganschow und M. Aslan.
Benutzung ist die Rückseite vollkommen abgenutzt.

Bibliographie: RPC I 2230. 2234. Taf. 99; Arslan 1992, Nr.15.

\section{Inv.-Nr. TR-00/4}

Nero, Nysa, AE, (vermutlich um 55 n. Chr.).

Av NEP $\Omega$ N KAI $\Sigma$ AP

Kopf des Nero nach $r$.

Rv NY $\Sigma$ A / E $\Omega$ N (in zwei Reihen über der Quadriga).

$\triangle \mathrm{IOMH} \triangle \mathrm{I} / \mathrm{ANOC}$ (in zwei Reihen unter der Quadriga).

Hades mit der geraubten Persephone auf galoppierender Quadriga nach $\mathrm{r}$.

Maße: Dm 1,85 cm, Stärke 0,35 cm, W 6,8 g

Vorderseite korrodiert. Grüne Patina.

Bibliographie: Regling 1913, 60; RPC I 2668

Taf.116.

6. Inv.-Nr. TR-00/01

Unbestimmt, AE.

Av Kaiserkopf n. links.

Maße: Dm 2,7 cm, Stärke 0,2 cm, W 10,6 g

Grüne Patina.

\section{Inv.-Nr. TR-00/9}

Constans, Antiocheia, AE, (337 - 340 n. Chr.).

Av [D N CONSTA]NS PF AVG

Kopf des Constans mit Lorbeerkranz nach r.

Rv GLORIA EXER[CITVS] SMN[ ]

Standarte zwischen bewaffneten Soldaten.

Maße: Dm 1,4 cm, D 0,15 cm, W 1,2 g

Guter Erhaltungszustand. Dunkelgrüne Patina.

Bibliographie: RIC VIII 471 Nr.12.

\section{Inv.-Nr. TR-00/6}

Iustinianus I, Konstantinopel, AE, (541/2 n. Chr.)

Av [D N IVSTINI] ANVS P P AVG

Frontalbüste des Iustinianus.

Rv 40 Nummi (M) Offizin $\Gamma$ r. X/4 im Abschnitt CON.

Maße: Dm 3,7 cm, Stärke 0,3 cm, W 22,6 g

Vorderseite korrodiert. Dunkle Patina.

Bibliographie: DOC I 88ff. Nr.40c.1 Taf. 27; MIB I 58f. Nr.95 Taf. 21; Wroth 1908, Nr.59. 60; Voegtli 1993, 55 Nr.765 Taf. 8.

9. Inv.-Nr. TR-00/13

Lichtteller eines Lampenständers.

Maße: Dm $7 \mathrm{~cm}, \mathrm{H}$ 6,4 cm (H mit dem erhaltenen Schaft 12,3 cm). 
Fragment eines bronzenen Kandelabers. Erhalten ist nur der bekrönende Teller mit einem vierkantigen Dorn zum Aufstecken einer Lampe oder Kerze. Kandelaberfuß, Lampe und untere Hälfte des Schaftes fehlen. Die Außenseite des tiefen Tellers ist mit zwei umlaufenden Ringen verziert. Dunkelgrüne Patina.

Datierung: Frühbyzantinisch (5./6. Jh. n. Chr.). Bibliographie: $\mathrm{Zu}$ ähnlichen Beispielen: Bailey 1996, 105 Q3923-Q3929 Taf.135-139; Wamser - Zahlhaas 1998, 80ff. Nr.75. 77. 79. 83; Davidson 1952, Nr.863 Taf.63; Waldbaum 1983, 104ff. Nr.614-616.

\section{Inv.-Nr. TR-00/11}

Gürtelschnalle vom Typ „Syrakus“"13.

Maße: erh. L 3,7 cm, B des Beschlages 2,5 cm, Stärke $0,3 \mathrm{~cm}$.

Fragmentierte Bronzeschnalle mit festem, herzförmigem Beschlag und kleinem Endknopf, verziert mit zwei mitgegossenen, gegenständigen Halbpalmetten. Entspricht nach Eger der Grundform des Typs „Syrakus“; auf der Unterseite des Beschlags zwei senkrechte Stiftösen. Im Beschlag Loch zum Einhängen des Dorns. Schnallenbügel mit anschließendem Teil des Beschlages abgebrochen; Dorn verloren. Grüne Patina.

Datierung: Frühbyzantinisch (spätes 6. - Mitte 7. Jh. n. Chr.).

Bibliographie: Schulze-Dörrlamm 2002, 171179; Eger 1996, 343ff.; Riemer 1995, 778ff. Abb. 13-17; Haas - Schewe 1993, 255ff.; Davidson 1952, 271 Nr.2185 Taf.114.

\section{Inv.-Nr. TR-00/10}

Zwiebelknopffibel vom Typ „Keller 4 C“ / „Pröttel 3/4 Var. D“. Evtl. aus östlicher Produktion.

Maße: erh. L. 4,2 cm (Länge Fuß 2,7 cm, Länge Bügel 2,2 cm), H 2,5 cm, Dicke der Bügel 0,6 $\mathrm{cm}$, Dicke des Fußes $0,8 \mathrm{~cm}$.

Fragmentierte Zwiebelknopffibel aus Bronze mit parallelseitigem Fuß, der länger als der Bügel ist; $\mathrm{Fu} ß$ ist breiter als der Bügel; an der Oberseite des Bügels zweireihige Kreisaugenverzierung, die im mittleren Teil des Bügels durch Abnutzung nicht mehr sichtbar ist; der Fuß ist mit trapezförmigen Kerben verziert (Fußverzierung d2 nach Swift);

${ }^{13}$ Werner 1955, 36ff. Abb. 2 Taf. 5,8-16.
Querarm mit Nadelkonstruktion und Zwiebelknöpfen verloren. Grüne Patina.

Datierung: 330-400 n. Chr.

Bibliographie: Keller 1971, 38-41; Pröttel 1988, 347-373; Swift 2000, 13-88; Waldbaum 1983, 116 Nr.683 Taf. 43.

\section{Inv.-Nr. TR-00/15}

Verkleidungsklammer vom Typ „Sardis 1“.

Maße: L 12,3 cm, Spitzenbreite 0,6 cm, B 1,5 $\mathrm{cm}$, Stärke 0,4 cm.

Flach-bandförmiger Zapfen, von der Spitze zum Ende hin breiter werdend. Nach Angaben der Restauratorin A.E. Gültekin handelt es sich um hartes, stahlähnliches Eisen. Die ursprünglich um $90^{\circ}$ gebogene Spitze fehlt. Man verwendete solche klammerartigen Zapfen zur Befestigung (Vertafelung) von Marmorplatten an Wänden. Er gehört dem in Sardis belegten ersten Typ an und datiert dort in spätrömische Zeit. Beidseitig korrodiert. Dunkelgrüne Patina.

Datierung: spätrömisch.

Bibliographie: Waldbaum 1983, 66f. Taf. 19.266-274; Boehringer-Krauss 1937, 125 Taf. $60 \mathrm{e} 7,9$.

\section{Inv.-Nr. TR-00/12}

Bronzeblech mit Punzverzierung.

Maße: Dm 8,9 cm, H 8,7 cm, Stärke 0,1 cm.

Dünnes, pilzförmiges Bronzeblech. Der äußere Rand ist mit fortlaufenden kreisrunden Punzen verziert, im Innenbereich drei „Punktrosetten“, mit dem gleichen Stempel wie die Randverzierung geschlagen. Spuren einer Befestigung oder Aufhängung sind nicht zu erkennen. Im Innenbereich unregelmäßige Löcher, Enden zueinandergebogen. Funktion unbekannt. Dunkelgrüne Patina.

Datierung: Frühbyzantinisch ?

Ausgehend von der pergamenischen Münze aus dem Jahr vor 133 v. Chr. streuen die Münzen über Augustus (27 v. Chr.), Nero (55 n. Chr.), Constans (337-340 n. Chr.) und Iustinian I. (541-2 n. Chr.) von hellenistischer bis in frühbyzantinische Zeit und decken so einen für Fundkomplexe von Bronzemünzen ungewöhnlich langen Zeitraum ab. Da die Cavea und das Bühnenhaus sowohl in späthellenistisch- 
frühaugusteischer Zeit als auch im 2. Jh. n. Chr. umgebaut wurden, kann man die pergamenische Münze sicher nicht mit der frühen Benutzungsphase des Theaters im 2. Jh. v. Chr. in Verbindung bringen. Obwohl die Stadt eine hellenistische Gründung ist, konnten bisher weder im Theater noch an anderen Stellen der Stadt Baustrukturen des 3. oder 2. Jhs. v. Chr. festgestellt werden. Der jetzige Stand der Grabung im Hyposkenion und im Kellergeschoss lässt ebenfalls keine ältere Bauphase des Bühnenhausfundamentes erkennen. Die alte Münze gehört daher auch zu dem Bronzedepot. Dies vor allem auch deswegen, weil sich unter den Funden auch ein runder Deckel aus Bronze befindet (Abb. 1), in den die pergamenische Münze genau passt. Reste einer im Deckel erhaltenen Eisenklammer und von Blei lassen darauf schließen, dass die hellenistische Münze mit dem Asklepioskopf als Verzierungselement in diesen Deckel eingesetzt war. Es ist davon auszugehen, dass die Münze als Altstück zur Deckelverzierung herangezogen wurde. Wann dies allerdings geschehen ist, lässt sich nicht mehr feststellen.

Der hellenistischen Münze folgen zwei Münzen von Augustus (27 - 23 v. Chr.) und je eine Münze von Nero (55. n. Chr.), Constans (337-340 n. Chr.) und Iustinianus I. (541/2 n. Chr.). $\mathrm{Zu}$ den Münzen treten eine Fibel aus dem 2. oder 3. Drittel des 4. Jhs. n. Chr., der Lichtteller eines Lampenständers aus dem 5./6. Jh. n. Chr., eine Gürtelschnalle aus dem späten 6. Jh. bzw. der 1. Hälfte des 7. Jhs., eine Verkleidungsklammer und ein punzverziertes, pilzförmiges Bronzeblech unbekannter Funktion.

Das Fragment einer bronzenen Fibel gehört zur Gruppe der spätantiken Zwiebelknopffibeln. Diese Fibeln sind ein charakteristischer Bestandteil der spätrömischen männlichen Amtskleidung.
Nach Darstellungen auf Mosaiken, Diptychen und Grabsteinen dienten sie als Mantelverschluss und wurden mit nach oben gerichtetem Fuß auf der rechten Schulter getragen. Die Fibeln wurden aus Bronze, Silber und Gold hergestellt und waren so Anzeiger der sozialen Differenzierung ihrer Träger. Das Exemplar aus Nysa ist der Gruppe „Pröttel 3/4 D“ zuzuordnen, die nach Pröttel in die Jahre zwischen 330 und 400 n. Chr. datiert ${ }^{14}$. Die Fibel ist demnach auf jeden Fall schon als Altstück im Theater von Nysa abgelagert worden, selbst wenn weitere Studien eine verlängerte Laufzeit des Typs bis in das 5. Jh. hin nachweisen könnten.

Bronzene Zwiebelknopffibeln treten vor allem im nordwestlichen Teil des spätantiken römischen Reiches häufig auf und sind in großer Anzahl hergestellt und verteilt worden. E. Swift hat festgestellt, dass viele Varianten des Typs 3/4 in Pannonien produziert worden sein müssen ${ }^{15}$. Das Exemplar aus Nysa hat mit einer doppelten Reihe von Kreisaugen auf dem Bügel und trapezförmigen Kerben auf dem Fuß (Fußverzierung d2 nach Swift) jedoch keine direkten Parallelen im Westen ${ }^{16}$. Daher stammt die Fibel aus Nysa mit hoher Wahrscheinlichkeit aus östlicher Produktion. Eine Verifizierung dieser Vermutung bedarf allerdings einer systematischen Sammlung und Untersuchung von Zwiebelknopffibeln im Osten des Reiches, wie sie E. Swift für den Westen unternommen hat. Der Fund aus Nysa zeigt trotz der geringfügigen Unterschiede in der Verzierung vor allem auch die großräumige Einheitlichkeit spätrömischer Amtsbekleidung und weist gemeinsam mit anderen östlichen Exemplaren darauf hin, dass in Massenproduktion gefertigte bronzene

\footnotetext{
${ }^{14}$ Pröttel 1988, 359-364.

${ }^{15}$ Swift 2000, 78.

16 Dank an Dr. Ellen Swift (Canterbury), die die Dekoration des Fundes aus Nysa freundlicherweise mit den von ihr gesammelten westlichen Exemplaren verglichen hat.
} 
Zwiebelknopffibeln kein Merkmal der nordwestlichen Provinzen darstellen. Der extreme zahlenmäßige Unterschied bekannter Fibeln zwischen West und Ost muss vor allem mit unterschiedlichen Forschungstraditionen und -interessen sowie dem daraus resultierenden Publikationsstand erklärt werden.

Der Lichtteller gehört zu einem Bronzekandelaber, auf dem nach Aussage spätantiker Buchillustrationen und Grabmalereien sowohl bronzene Öllampen als auch Kerzen aufgesteckt werden konnten. Die verlorene Basis hatte nach vergleichbaren Funden wohl die Form eines nach unten gerichteten Blütenkelches mit drei tatzenförmigen Füßen. Mit der Basis, dem wohl aus mehreren Teilen zusammengesetzten Ständer und dem Lichtteller besaß der Kandelaber wahrscheinlich eine Höhe von 20 bis $50 \mathrm{~cm}$, wobei gelegentlich auch höhere Exemplare vorkommen ${ }^{17}$. Lampen und Lampenständer wie dieser nahmen unter den spätantiken Alltagsgeräten einen besonderen Raum ein. Man benötigte sie im privaten Bereich genau wie in öffentlichen Gebäuden, in frühchristlicher Zeit dann vor allem auch in Kirchen, in denen sie in großer Zahl aufgestellt waren. Wie die anderen Gegenstände war auch dieser Kandelaber schon zerstört, als er im Theater von Nysa versteckt wurde.

Verkleidungsklammern werden häufig in öffentlichen Gebäuden gefunden, deren Wände mit Marmorplatten verziert waren. Ihre um $90^{\circ}$ gebogene kurze Spitze hielt die Marmorplatte von unten, während der Schaft hinter der Platte verankert war. J. Waldbaum hat die Funde solcher Klammern aus Sardis in zwei Typen unterteilt. Der Fund aus Nysa entspricht dem ersten Typ nach Waldbaum, der in Sardis allgemein in die spätrömische

\footnotetext{
${ }^{17}$ Mit zahlreichen Vergleichsbeispielen: V.H. Ebern u.a. in: Wamser - Zahlhaas 1998, 80-88.
}

Zeit datiert wird ${ }^{18}$. Der Altmetallsammler des 7. Jhs. hat dieses Stück wahrscheinlich in einem der öffentlichen Bauten in Nysa gefunden, der sich im 7. Jh. schon im Zustand des Verfalls befunden haben muss.

Die Gürtelschnalle gehört einem häufigen und weit verbreiteten Typ an, für den J. Werner 1955 die Bezeichnung „Typ Syrakus" einführte ${ }^{19}$. C. Eger untergliederte diese Schnallen, indem er einen Grundtyp, zwei Varianten und zwei Imitationen definierte $^{20}$. Das Exemplar aus Nysa entspricht dem Grundtyp mit einander zugewandten Halbpalmetten und „Seitentrieben“ seitlich der Dornbefestigung. Diese kleinen Bronzeschnallen mit festem herzförmigem Beschlag, Palmettenverzierung, am Stück aus Nysa zu ergänzendem, flach-ovalem Bügel mit eingetiefter Dornrast und Höckerdorn wurden an einem schmalen Ledergürtel von Männern, Frauen und Kindern getragen. Mit Hilfe geschlossener Grabfunde kann dieser Schnallentyp in das späte 6 . Jh. bis um die Mitte des 7. Jhs. datiert werden ${ }^{21}$. E. Riemer vermutete in Bezug auf das Männergrab 53 von Piana degli Albanesi (Sizilien), in dem zusammen mit einer Schnalle vom Typ „Syrakus“ unter anderem auch eine nordafrikanische Tonlampe vom nur bis zum Ende des 5. Jh. hergestellten Typ Hayes IIA $^{22}$ gefunden wurde, eine frühe Entstehung des Typs „Syrakus“ im beginnenden 6. Jh. ${ }^{23}$. Mit einem Hinweis auf die dann für ein solches Kleidungsaccessoire ungewöhnlich lange Laufzeit von über 150 Jahren und fehlende Exemplare in byzantinischen Donaukastellen, die im späten $6 . \quad \mathrm{Jh}$. aufgegeben wurden, lehnt M. Schulze-

\footnotetext{
${ }^{18}$ Waldbaum 1983, 66f. Taf. 19.266-274.

19 Werner 1955, 36ff. Abb. 2 Taf.5,8-16.; SchulzeDörrlamm 2002, 171-179 fasst die gleichen Schnallen als „Typ D12“ zusammen.

${ }^{20}$ Eger 1996, 344.

${ }^{21}$ Schulze-Dörrlamm 2002, 179.

${ }^{22}$ Hayes 1972, $311 \mathrm{ff}$.

${ }^{23}$ Riemer 1995, 778ff. Abb. 13-17, hier 779 Abb. 1.
} 
Dörrlamm diesen Datierungsvorschlag Riemers jedoch $\mathrm{ab}^{24}$. Außer einem durch die jüngste Münze des Befundes definierten terminus post quem von 541/2 n. Chr., dem in Anbetracht der durch die Münzen dieses Befundes abgedeckten großen Zeitspanne keine allzu große Bedeutung beizumessen ist, kann das Exemplar aus Nysa keine neuen Erkenntnisse zur Datierungsproblematik liefern.

Trotz einer damals erheblich geringeren Materialbasis, hatte J. Werner diese Schnallen 1955 schon richtig als „Massenartikel in stereotyper Ausfertigung“" beschrieben ${ }^{25}$. Im Gegensatz zu älteren Arbeiten, die die Schnallen wegen ihrer Häufigkeit in Gräberfeldern in den nördlichen Grenzgebieten des Reiches barbarischem Kunsthandwerk zuordneten, betonte Werner den ,byzantinischen' Ursprung dieser Stücke. Ohne die begriffliche Bedeutung von ,byzantinisch' näher zu erläutern, verwies Werner vor allem auf die charakteristische Pflanzenornamentik als Anzeiger einer mediterranen Herkunft der Schnallen. Gerade aus dem Herzen des byzantinischen Reiches waren zu dieser Zeit aber noch sehr wenige Funde bekannt, so dass diese Interpretation damals durch die Verbreitungskarten noch nicht gestützt wurde. Durch sorgfältige Ausgrabungen und vor allem durch die Publikation von Kleinfunden sind in den letzten Jahrzehnten jedoch so viele Siedlungsfunde bekannt geworden, dass an der weiten Verbreitung dieses Typs im Mittelmeerraum keine Zweifel mehr bestehen. In der aktuellsten Verbreitungskarte der Schnallen vom Typ Syrakus finden sich in der Türkei mit İstanbul, Sardis und Anemurium jedoch nur drei sicher $\mathrm{zu}$ lokalisierende Fundstellen ${ }^{26}$. Mit unserem Neufund aus Nysa und weiteren, bei

\footnotetext{
${ }^{24}$ Schulze-Dörrlamm 2002, 179.

${ }^{25}$ Werner 1955, 37.

${ }^{26}$ Schulze-Dörrlamm 2002, 176 Abb. 62.
}

Schulze-Dörrlamm noch nicht erfassten Exemplaren aus Amorium (Emirdağ), Istanbul, Seleucia Trachea (Meryamlı/ Silifke) und Antiocheia (Hatay), auf die M. Lightfoot hinweist ${ }^{27}$, füllt sich die Verbreitungskarte nun allmählich auch für die Türkei. Zählt man die vielen Schnallen ohne sicheren Fundort in Sammlungen hinzu - alleine im RGZM Mainz tragen 14 Exemplare die nicht näher einzugrenzende Herkunftsangabe „Kleinasien“ - so gleicht sich das bisherige Übergewicht des westlichen Mittelmeerraumes gegenüber den östlichen Regionen aus (Abb. 13).

Eine Lokalisierung des oder der Produktionsorte der Schnallen wird hierdurch nicht einfacher. Die Schnallen wurden in einem Gussverfahren mit verlorener Form hergestellt ${ }^{28}$. Spuren dieses Produktionsverfahrens und damit Hinweise auf Produktionsorte konnten bisher nicht festgestellt werden. Daher bleibt offen, ob sich diese Schnallen durch Handel, durch andere persönliche Kontakte oder durch Herstellung an vielen verschiedenen Orten ausgebreitet haben. Unzweifelhaft zeigt die weite Verbreitung dieser Schnallen jedoch ein in diesem Detail erstaunlich einheitliches, vom byzantinischen Mittelmeerraum ausgehendes Modeverständnis in frühbyzantinischer Zeit.

\section{Schlussfolgerung}

Abgesehen von der hellenistischpergamenischen Münze stammen die Funde, die vom letzten Viertel des 1. Jhs. v. Chr. bis in die Mitte des 7. Jhs. n. Chr. streuen, aus dem Nutzungszeitraum des Theaters von Nysa am Mäander ${ }^{29}$. Mit den epigraphischen Belegen und den bescheidenen baulichen

27 Lightfoot 2003, im Druck. Die dort erwähnte Schnalle aus Pergamon, die A. Conze 1913 publiziert hat, ist keine Schnalle vom Typ "Syrakus".

${ }^{28}$ Haas - Schewe 1993, $257 \mathrm{ff}$.

${ }^{29}$ Kadioğlu 2002, 247. 
Veränderungen in frühbyzantinischer Zeit ${ }^{30}$ belegen auch die Bronzefunde die lange Benutzung des Baus bis in das 7. Jh. n. Chr. $\mathrm{Ob}$ der schon verfallende Bau zu dieser Zeit jedoch noch im Rahmen seiner ursprünglichen Funktion genutzt wurde, ist unklar.

Die lange, durch die Funde abgedeckte Zeitspanne wirft auch Fragen nach der Aussagekraft der Funde in Bezug auf die bauhistorische Entwicklung des Theaters auf. Gerade die alten Fundstücke aus hellenistischer Zeit und dem 1. Jh. n. Chr. hatten $\mathrm{zu}$ der Vermutung geführt, das augusteische Fundament sei beim Wiederaufbau der scaenae frons im 2. Jh. $\mathrm{n}$. Chr. eventuell nicht verändert worden. Die Arbeiten der Kampagne 2003, bei denen der Korridor und die Gewölbe des Hyposkenions vollständig freigelegt wurden, haben jedoch gezeigt, dass das Fundament des Bühnenhauses im 2. Jh. n. Chr. einheitlich errichtet worden ist und nur das Fundament des dritten Podiums aus dem Rahmen fällt. In diesem Fundament wurden zwei mit je drei Faszien verzierte Architravblöcke aus Kalkstein verbaut, die sich aufgrund ihres Aufbaues deutlich von den anderen Architravblöcken unterscheiden. Es ist daher davon auszugehen, dass diese älteren Architravblöcke beim Wiederaufbau der scaenae frons als Spolien verbaut worden sind. Eine gemeinsame Niederlegung des gesamten Fundkomplexes ist damit noch wahrscheinlicher geworden. Als Bronzeschrottdepot des 7. Jhs. hat der Fund aber keine weitere Aussagekraft für den Bau selbst, mit Ausnahme der Tatsache, dass er in dieser Zeit noch begangen wurde, um die gesammelten Bronzen dort $\mathrm{zu}$ verbergen. Zur Herkunft der Stücke können keine Aussagen getroffen werden. Es ist aber davon auszugehen, dass der Sammler sie in Nysa gefunden hat, da sich der Transport

${ }^{30}$ Kadıŏglu 2002, 244ff. dieser unbrauchbaren Stücke über weitere Strecken sicher nicht gelohnt hat.

Unter den Funden selbst tragen vor allem die Gürtelschnalle vom Typ „Syrakus“ und die Zwiebelknopffibel zur Kenntnis der Verbreitung dieser Typen im Osten des Reiches bei.

Dr. Musa Kadıŏlu, Ankara Üniversitesi, Dil ve Tarih-Coğrafya Fakültesi, Arkeoloji Bölümü 06100 Sıhhiye / Ankara - Türkei.

e-mail: kadioglu@humanity.ankara.edu.tr

Philipp von Rummel M.A., Albert-LudwigsUniversität Freiburg, Institut für $\mathrm{Ur}$ - und Frühgeschichte und Archäologie des Mittelalters, Belfortstr. 22, 79085 Freiburg im Breisgau Deutschland.

e-mail: rummel@ufg.uni-freiburg.de

\section{Liste 1: Schnallen vom Typ Syrakus ${ }^{31}$}

1. Edfu (M.-J. Chavane, Salamine de Chypre VI: Les petits objets (1975) 163 mit Anm. 2).

2. „Ägypten” (F. Petrie, Objects of daily use, British School of Archaeology in Egypt 42 (1927) 23 Nr. 15, Taf. XVIII 15).

3. Byblos oder Tyros (B. Haas, AnzGermNatMus 1993, 264 Nr. 2 Abb. 1).

4. „Iran“: 2 Ex. (Schulze-Dörrlamm 2002, Kat.Nr. 144. 154).

5. Nalčik: 2 Ex. (Očet Imperatorskoj Arch. Komm. 1897 (1900), 44 Abb. 122; T.J. Arne, La Suède et l'Orient (1914) 143).

6. Djurso bei Novorossijsk: 13 Ex. aus Gräbern (unpubliziert).

7. Borisovo bei Novorossijsk, Grab 138 (V. Sakhanioff, Izv. Imperatorskoj Arch. Komm. 56 (1914) 75ff.; I. Erdelyi, Az avarsag es kelet a Regeszeti Farrasok Türkeben (1982) Taf. 6).

8. Taman (J. Werner, KölnJb 1 (1955) 46 Nr. 7).

9. Kertsch: 6 Ex. (British Museum - a guide to the Anglo-Saxon and foreign Teutonic antiquities in the Department of British and Medieval

${ }^{31}$ Die Fundliste wurde mit Ausnahme der Nummern 76-82 von Riemer und Schulze-Dörrlamm übernommen und hier noch einmal detailliert aufgeführt, um den Zugang zur Literatur zu erleichtern. 
Antiquities (1923) 60, 170; D. Csallány, ActaAntHung 2 (1954) 344 Taf. II 9; J. Werner, KölnJb 1 (1955) 46 Nr. 6; A. MacGregor, Ashmolean Museum Oxford. A Summary catalogue of the Continental Archaeological Collections (1997) 241 Nr. 1909. 805).

10. Feodosia (C. Eger, MAIE Tavrii 5 (1996) 343).

11. Aromat: 6 Ex. (I.I. Loboda, SovA 1976, 2, 135ff. Abb. 4, 2-5; ebd., Abb. 5, 23-24).

12. Bakta (V.B. Kovalevskaja, Eurasische Gürtelschnallengarnituren des 4.-9. Jahrhunderts (1979) Taf. VIII 11).

13. Brodovski (V.B. Kovalevskaja, Eurasische Gürtelschnallengarnituren des 4.-9. Jahrhunderts (1979) Taf. VIII 12).

14. Čufut Kale, Grabkammer 7 (A.I. Aibabin, Pogrebenija konza VII-pervoj poloviny VIII v.v. Krymu. In: Drevnosti epochy velikogo pereselenija narodov V-VIII vekov (1982) Abb. $1,11)$.

15. Eski Kermen, Grab 316 (A.I. Aibabin, MAIE Tavrii 2 (1991) 43ff. Abb. 2, 3).

16. Gursuff (D. Csallány, ActaAntHung 2 (1954) 344; J. Werner, KölnJb 1 (1955) 45 Nr. 5a).

17. Lučistoe, Kammer 10: 3 Ex. (A.I. Aibabin, MAIE Tavrii 5 (1995) Abb. 6, 2. 4-5).

18. Sacharna Golivka: 2 Ex. (Archaeologicni Pamatki URSR 13 (1963) 53 Abb. 11, 1; V.B. Kovalevskaja, Eurasische Gürtelschnallengarnituren des 4.-9. Jahrhunderts (1979) Taf. VIII 13).

19. Skalistoe, Gräber 258, 278, 320, 471: 4 Ex. (E.V. Vejmarn - A.I. Aibabin, Skalistinstij Mogil'nik (1993) 47 Abb. 28,8. 51 Abb. 31,5. 70 Abb. 47, 12. 118 Abb. 85, 4).

20. Suuk Su, Gräber 29, 32, 58, 85: 6 Ex. (N. Riepnikoff, Izv. Imperatorskoj Arch. Komm. 19 (1906) 1ff.; D. Csallány, ActaAntHung 2 (1954) 344; J. Werner, KölnJb 1 (1955) 45 Nr. 5).

21. Chersones: 16 Ex. (A.L. Jakobson, Das frühmittelalterliche Cherson (1959) 273 Abb. 139, 1-2; A.I. Aibabin, SovA 1982, 3, $190 \mathrm{ff}$. Abb. 1, 5; T.J. Arne, La Suède et l'Orient (1914) 142f.).

22. Salamis (M.-J. Chavane, Salamine de Chypre VI: Les petits objets (1975) 162f. Nr. 466 Taf. 46, 466. Taf. 69, 466).

23. Anemurium : 2 Ex. (Russell 1982, 133ff., 152 Anm. 36 Abb. 7, 14-16).
24. Sardis, Akropolis und Gebäude B: 2 Ex. (Waldbaum 1983, 118 Nr. 689, 690 Taf. 44, 689, 690).

25. Istanbul: 4 Ex. (D. Csallány, ActaAntHung 2 (1954) 344 Taf. III 1-2; aus einer Privatsammlung: K. Bittel - A.M. Schneider, $A A$ 1944-45 (1949) 78 Nr. 3 Taf. 27, 3-4; J. Werner, KölnJb 1 (1955) 45 Nr. 4; Saraçhane: M.A.V. Gill, Small Finds, in: R.M. Harrisson (Hrsg.), Saraçane I (1991) 265 Nr. 561 Abb. 402).

26. „Kleinasien“: 14 Ex., genauer Fundort unbekannt, heute im RGZM Mainz (SchulzeDörrlamm 2002, Kat. Nr. 145-150. 152-153; T.J.

Arne, La Suède et l'Orient (1914) 143).

27. Samos : 3 Ex. (U. Jantzen u.a., $A A$ 1975, 32 Abb. 20 ; W, Martini - C. Steckner, Das Gymnasium von Samos. Das frühbyzantinische Klostergut. Samos XVII (1993) Abb. 36, 6; 37, 3; $38,4)$.

28. Athen, Agora Grab 10 (K.M. Setton, Speculum 25 (1950) 522; J. Werner, KölnJb 1 (1955) 45 Nr. 1)

29. Daskaleio auf Romvi (A. Avraméa, Byzantina Sorboensia 15 (1997) 90 Taf. IVa, 1).

30. Korinth: 2 Ex. (Davidson 1952, 271 Nr. 2185 Taf. 114, 2185; J. Werner, KölnJb 1 (1955) 45 Nr. 2; Grabfund bei der Basilika von Kraneion: D. Pallas, Byzantino-Bulgarica 7 (1981) 298f. Abb. 5).

31. Plateia (A. Avraméa, Byzantina Sorboensia 15 (1997) 90 Taf. IVc, 1).

32. Edessa (F. Petsas, Adelt 24 (1969) 305ff. Taf. 320; V. Popović, CRAI 1980, 230ff. Abb. 1; D. Pallas, Byzantino-Bulgarica 7 (1981) Abb. 4).

33. „Griechenland“ (J. Werner, KölnJb 1 (1955) 45 Nr. 3).

34. Sofia: 2 Ex. (British Museum - a guide to the Anglo-Saxon and foreign Teutonic antiquities in the Department of British and Medieval Antiquities (1923) 60. 170; J. Werner, KölnJb 1 (1955) 46 Nr. 10).

35. Balta Verde, Grab 1 (D. Berciu - E. Comsa, Mat. si Cerc. Arh. 2 (1956) 403 Abb. 132, 1; U. Fiedler, Studien zu den Gräberfeldern des 6. bis 9 Jahrhunderts an der unteren Donau (1992) 88ff. Abb. 14).

36. Csákberény, Grab 215 (N. Fettich, Das awarenzeitliche Gräberfeld von PilismarotBasaharc (1965) 109 Abb. 176, 8 und 8a).

37. Dunapentele (D. Csallány, ActaAntHung 2 (1954) 344; J. Werner, KölnJb 1 (1955) 46 Nr. 14). 
38. Százhalombatta (I. Dienes u.a., Pest megye régészti Topográfája (1986) 243 Taf. 38, 19).

39. Szeged-Fehérto $A$, Grab 34 (D. Csallány, ActaAntHung 2 (1954) 344 Taf. III 3 und VII 1; J. Werner, KölnJb 1 (1955) 46 Nr. 13).

40. Szelevény (A. Marosi - N. Fettich, Trouvailles avares de Dunapentele (1936) 69ff. Abb. 27, 4 ; D. Csallány, ActaAntHung 2 (1954) 344 Taf. III 5; J. Werner, KölnJb 1 (1955) 46 Nr. 12).

41. Kölked-Feketekapu, Grab 325 (A. Kiss, das awarenzeitlich-gepidische Gräberfeld von Kölked-Ferketekapu A (1996) 207 Taf. 69, 325, 2).

42. Salona (J. Werner, KölnJb 1 (1955) $46 \mathrm{Nr}$. 11; Z. Vinski, VjesAMuzZagreb III 3 (1967) Taf. XVI 5).

43. Zadar (Z. Vinski, VjesAMuzZagreb III 3 (1967) Taf. XVI 4).

44. „Pula“, im Museum Pula mit unbekanntem Fundort (J. Werner, KölnJb 1 (1955) 46 Nr. 11a; Z. Vinski, VjesAMuzZagreb III 3 (1967) Taf. XVI 7).

45. Celega bei Novigrad (B. Marušić, JadrZbor (1961-62) Taf. V 3; Z. Vinski, VjesAMuzZagreb III 3 (1967) Taf. XVI 6).

46. „Italien“: 4 Ex. (D. Csallány, ActaAntHung 2 (1954) 344 Taf. II 7-8; Taf. III 4; 6).

47. Brianza (D. Csallány, ActaAntHung 2 (1954) 344; J. Werner, KölnJb 1 (1955) 46 Nr. 19).

48. Cividale S. Giovanni, Grab 237 (Riemer 1996, 798 Nr. 1 - unpubliziert im Museum Cividale).

49.Voltago (N. Åberg, Die Goten und Langobarden in Italien (1923) 155; J. Werner, KölnJb 1 (1955) 46 Nr. 16).

50. Luni (O. von Hessen, Secondo contributo all'archeologia longobarda in Toscana. Reperti isolati e di provenienza incerta (1975) 82 Nr. 22 Taf. 25,5).

51. Umgebung von Ravenna (M.G. Maioli, Felix Ravenna 111-112 (1976) 109f. Abb. 16).

52. Perugia (D. Csallány, ActaAntHung 2 (1954) 344; J. Werner, KölnJb 1 (1955) 46 Nr. 18).

53. Nocera Umbra, Grab 156 (A. Pasqui - R. Paribeni, MonAnt 25 (1918) 339f.; N. Åberg, Die Goten und Langobarden in Italien (1923) 114 Abb. 206; J. Werner, KölnJb 1 (1955) 46 Nr. $15)$.

54. Rom (F. Paolucci, Museo Nazionale del Bargello. Kat. Florenz (1994) 80).
55. Mattinata (C. d'Angela, Stud. Medievali Ser. III, 27 (1986) 915 Nr. 4; C. d'Angela, Taras IX/1-2 (1989) 131 Taf. L 12).

56. Neapel (D. Csallány, ActaAntHung 2 (1954) 344 Taf. III 7; J. Werner, KölnJb 1 (1955) 46 Nr. 19a; W. Menghin, Gotische und langobardische Funde aus Italien (1983) 68 Nr. 45 Taf. 14, 45; B. Haas, AnzGermNatMus 1993, 264 Nr. 1 Abb. $1)$.

57. Rutigliano, Grab 6/1983-84: 2 Ex. (C. d'Angela, Taras IX/1-2 (1989) 119ff. Taf. L 1011 ; C. d'Angela - G. Volpe, MEFRA 103-2 (1991) 785ff. Abb. 34).

58. „Süditalien“, genauer Fundort unbekannt (D. Csallány, ActaAntHung 2 (1954) 344).

59. Piana degli Albanesi, Grab 53 (Di terra in terra - Nouve scoperte archeologiche nella provincia di Palermo. Kat. Palermo (1993) 174).

60. Syrakus-Buffaloro (P. Orsi, ByzZ 21 (1912) 203 D Abb. 23; P. Orsi, Sicilia bizantina (1942) 188f. D Abb. 94; J. Werner, KölnJb 1 (1955) 46 Nr. 20).

61. Tharros (L. Pani Ermini - M. Marinone, Museo archeologico nazionale di Cagliari. Catalogo dei materiali paleocristiani $e$ altomedievali (1981) 99 Taf. 149 Nr. 149 Abb. 149).

62. „Sardinien“, genauer Fundort unbekannt: 2 Ex., heute im Museo Archeologico Turin und im Museum Sassari (J. Werner, KölnJb 1 (1955) 46 Nr. 24; O. von Hessen, Byzantinische Schnallen aus Sardinien im Museo Archeologico zu Turin, in: G. Kossack - G. Ulbert (Hrsg.), Studien zur Vor- und Frühgeschichtlichen Archäologie. Festschrift J. Werner (1974) 554).

63. „Südspanien“, genauer Fundort unbekannt, heute im RGZM Mainz (Schulze-Dörrlamm 2002, Kat.Nr. 151).

64. Karthago: 2 Ex. (British Museum - a guide to the Anglo-Saxon and foreign Teutonic antiquities in the Department of British and Medieval Antiquities (1923) 60. 153; J. Werner, KölnJb 1 (1955) 47 Nr. 26; C. Eger, CEDAC Carthage 19 (1999) 13 Abb. 1-2).

65. Constantine, Rummel-Schlucht: 3 Ex. (M. Besnier - R. Blanchet, Collection Farges. Musées et collections archéologiques de l'Algérie et de la Tunisie 9 (1900) 63 Nr. 10-12 Taf. X 12-13; J. Werner, KölnJb 1 (1955) 47 Nr. 27).

66. Freilassing-Salzburghofen, Grab 68 (R. Knöchlein, Studien zur Archäologie der 
Merowingerzeit im Rupertiwinkel. Diss. München (1997) Taf. 6, B, 2).

67. Regensburg, St. Emmeram - Pfarrkirche St. Rupert, Frauengrab (Bay VgBl, Beih. 1 (1987) 168 Abb. 115, 3; U. Osterhaus, BerBayDenkmPfl 34/35 (1993/94) 202ff. Abb. 3).

68. Hahnheim (J. Werner, KölnJb 1 (1955) 47 Nr. 28; G. Zeller, MainzZ 67-68 (1972-73) 330ff. Abb. 12, 76).

69. Langenlonsheim, Grab 447 (B. Stümpel, MainzZ 76 (1981) 145ff. Abb. 4-447, 6).

70. Stockstadt (R. Koch, Die Bodenfunde der Völkerwanderungszeit aus dem Main-TauberGebiet (1967) 164 Abb. 11,7).

71. Belvaux (J. Werner, KölnJb 1 (1955) 47 Nr. 29).

72. Broyle bei Chichester (The Antiquaries Journal 4 (1924), 49f.).

73. Colchester (R. Hattatt, Ancient Brooches (1989) Nr. 254 Abb. 38).

74. „Kent“, genauer Fundort unbekannt (British Museum - a guide to the Anglo-Saxon and foreign Teutonic antiquities in the Department of British and Medieval Antiquities (1923) Taf.
XXXVIII 5; N. Åberg, The Anglo-Saxons in England during the Early Centuries after the Invasion (1926) 102 Abb. 186; J. Werner, KölnJb 1 (1955) 47 Nr. 30 Abb. 2, 3).

75. „Sussex“, genauer Fundort unbekannt, heute im Museum Lewes (N. Åberg, The Anglo-Saxons in England during the Early Centuries after the Invasion (1926) 207 Nr. 108; J. Werner, KölnJb 1 (1955) 47 Nr. 31).

76. Nysa am Mäander, Theater (s.o.)

77. Amorium/ Emirdağ (C.S. Lightfoot - E.A. Ivison, AnatSt 46 (1996) 101f. Abb. 5; Lightfoot 2003).

78. Seleucia Trachea/ Silifke-Meryamlık, AyaThekla-Kirche (G. Dagron - D. Feissel, Inscriptions de Cilicie (1987) 252f. Taf. LXIV 13).

79. Antiochia/ Hatay (Russell 1982, 152 Anm. 36)

80. Aïn Wassel bei Teboursouk (M. de Vos (Hrsg.) Rus Africum. Terra acqua olio nell'Africa settentrionale. Scavo e ricognizione nei dintorni di Dougga. Austellungskat. Trento (2000) Abb. 58,12 und 58, 14).

\section{Abbildungsverzeichnis}

Zeichnung 1: Vorderansicht des Lichttellers eines Lampenständers.

Zeichnung 2: Vorder- und Seitenansichten der Gürtelschnalle vom Typ "Syrakus".

Zeichnung 3: Vorder- und Seitenansichten der Zwiebelknopffibel.

Zeichnung 4: Vorder- und Seitenansichten des Verkleidungsklammer.

Zeichnung 5: Rekonstruierte Vorderansicht des punzverzierten Bronzeblechs.

Abbildung 1-7: Fotos der Fundmünzen.

Abbildung 8-12: Fotos der Bronzegegenstände.

Abbildung 13: Verbreitungskarte der Gürtelschnallen vom Typ Syrakus nach Schulze-Dörrlamm 2002, Abb. 62 und Riemer 1995, Fundliste I und Abb. 30 mit Ergänzungen türkischer Funde (Nr. 76 - 79) und einem afrikanischen Exemplar (81). Nummerierung nach Schulze-Dörrlamm, Fundortnachweise (siehe Liste 1) nach Riemer und Schulze-Dörrlamm mit Ergänzungen. 


\section{Abkürzungen und Bibliographie}

\author{
Ak1n 1968 \\ Arslan 1992 \\ Bailey 1996 \\ BMC Greek Coins \\ Boehringer - Krauss 1937 \\ Davidson 1952 \\ DOC I
}

Eger 1996

Haas - Schewe 1993

Hayes 1972

İdil 2002

Kadıoğlu 2002

Keller 1971

Lightfoot 2003

MIB I

Pröttel 1988

Regling 1913

RIC VIII

Riemer 1995

\section{RPC I}

Russel 1982

Schulze-Dörrlamm 2002

Sezer - Tuna - Peker 1988

Sezer - Tuna - Peker 1989

SNG v. Aulock 1957

Swift 2000

Voegtli 1993

Waldbaum 1983

Wamser - Zahlhaas 1998

Werner 1955

Wittek 1934

Wroth 1908
H. Akın, Aydın Oğulları Tarihi Hakkında Bir Araştırma (1968).

M. Arslan, Museum of Anatolian Civilizations. Roman coins (1992).

D.M. Bailey, A catalogue of the lamps in the British Museum (1996).

A Catalogue of the Greek Coins in the British Museum.

E. Boehringer - F. Krauss, Das Temenos für den Herrscherkult : 'Prinzessinnen Palais'. AvP 9 (1937).

G.R. Davidson, The minor objects. Corinth 12 (1952).

Catalogue of the byzantine coins in the Dumbarton Oaks Collection and in the Whittemore Collection, edited by A.R. Bellinger - Ph. Grierson I-III (1966-1973).

C. Eger, Eine byzantinische Gürtelschnalle von der Krim in der Sammlung des Hamburger Museums für Archäologie, Materialy po archeologii, istorii $i$ etnografii Tavrii 5 (1996) 344-348. 584f.

B. Haas - R. Schewe, Byzantinische Gürtelbeschläge im Germanischen Nationalmuseum, AnzGermNatMus 1993, 255-273.

J.W. Hayes, Late Roman Pottery (1972).

V. İdil, Nysa'da 2000 Y1lı Kazıları, KST 23.1 (2002) 77-86.

M. Kadıŏlu, Die scaenae frons des Theaters von Nysa am Mäander, Dissertation Albert-Ludwigs-Universität Freiburg (2002).

http://www.freidok.uni-freiburg.de/volltexte/531

E. Keller, Die spätrömischen Grabfunde in Südbayern (1971).

M. Lightfoot, Afyon Arkeoloji Müzesi ve Amorium Kazılarında Bulunan Bizans Kemer Tokolar1, TürkAED 3, 2002 (2003) 119-134 (im Druck).

W. Hahn, Moneta Imperii Byzantini. Von Anastasius I. bis Justinianus I. [491-565] (1973).

Ph. M. Pröttel, Zur Chronologie der Zwiebelknopffibeln, JbRGZM 35/1 (1988) 359364.

K. Regling, in: W. von Diest, Nysa ad Maeandrum. Nach Forschungen und Aufnahmen in den Jahren 1907 und 1909, JdI Ergh.10 (1913).

J.P.C. Kent, in: C.H.V. Sutherland - R.A.G. Carson (Hrsg.), Roman Imperial Coinage VIII (1981).

E. Riemer, Byzantinische Gürtelschnallen aus der Sammlung Diergardt im RömischGermanischen Museum Köln, KölnJb 28 (1995) 777-809.

A. Burnett - M. Amandry - P.R. Ripollès, Roman Provincial Coinage I. From the death of Caesar to the death of Vitellius [44 BC - AD 69] (1992).

J. Russel, Byzantine Instrumenta Domestica from Anemurium: the significance of context, in: R.L. Hohlfelder (Hrsg.), City, Town, and Countryside in the Early Byzantine Era (1982).

M. Schulze-Dörrlamm, Byzantinische Gürtelschnallen und Gürtelbeschläge im Römisch-Germanischen-Zentralmuseum I (2002).

V. Sezer - M. Tuna - R. Peker, Nysa Tiyatrosu Dış Cephe ve Doğu-Batı Girişleri Açma Çalışmaları, TürkAD 27, 1988, 85-100.

V. Sezer - M. Tuna - R. Peker, Nysa Tiyatrosu'nda Skene Kazısı ve Podyumlar, TürkAD 28, 1989, 307-322.

Sylloge Nummorum Graecorum Deutschland. Sammlung von Aulock: Mysien H. 4 (1957).

E. Swift, Regionality in dress accessories in the late Roman West (2000).

H. Voegtli, Die Fundmünzen aus der Stadtgrabung von Pergamon, PF 8 (1993).

J.C. Waldbaum, Metalwork from Sardis: the finds through 1974 (1983).

L. Wamser - G. Zahlhaas, Rom und Byzanz: Archäologische Kostbarkeiten aus Bayern. Katalog zur Ausstellung der Prähistorischen Staatssammlung München (1998).

J. Werner, Byzantinische Gürtelschnallen des 6. und 7. Jahrhunderts aus der Sammlung Diergardt, KölnJb 1 (1955) 36-48.

P. Wittek, Das Fürstentum Mentesche. Studie zur Geschichte Westkleinasiens im 13. - 15. Jh,. IstMitt (1934); ders. Menteşe Beyliği ${ }^{3}$ (türkische Übersetzung von O.Ş. Gökyay) [1999].

W. Wroth, BMC Imperial Byzantine Coins I (1908). 

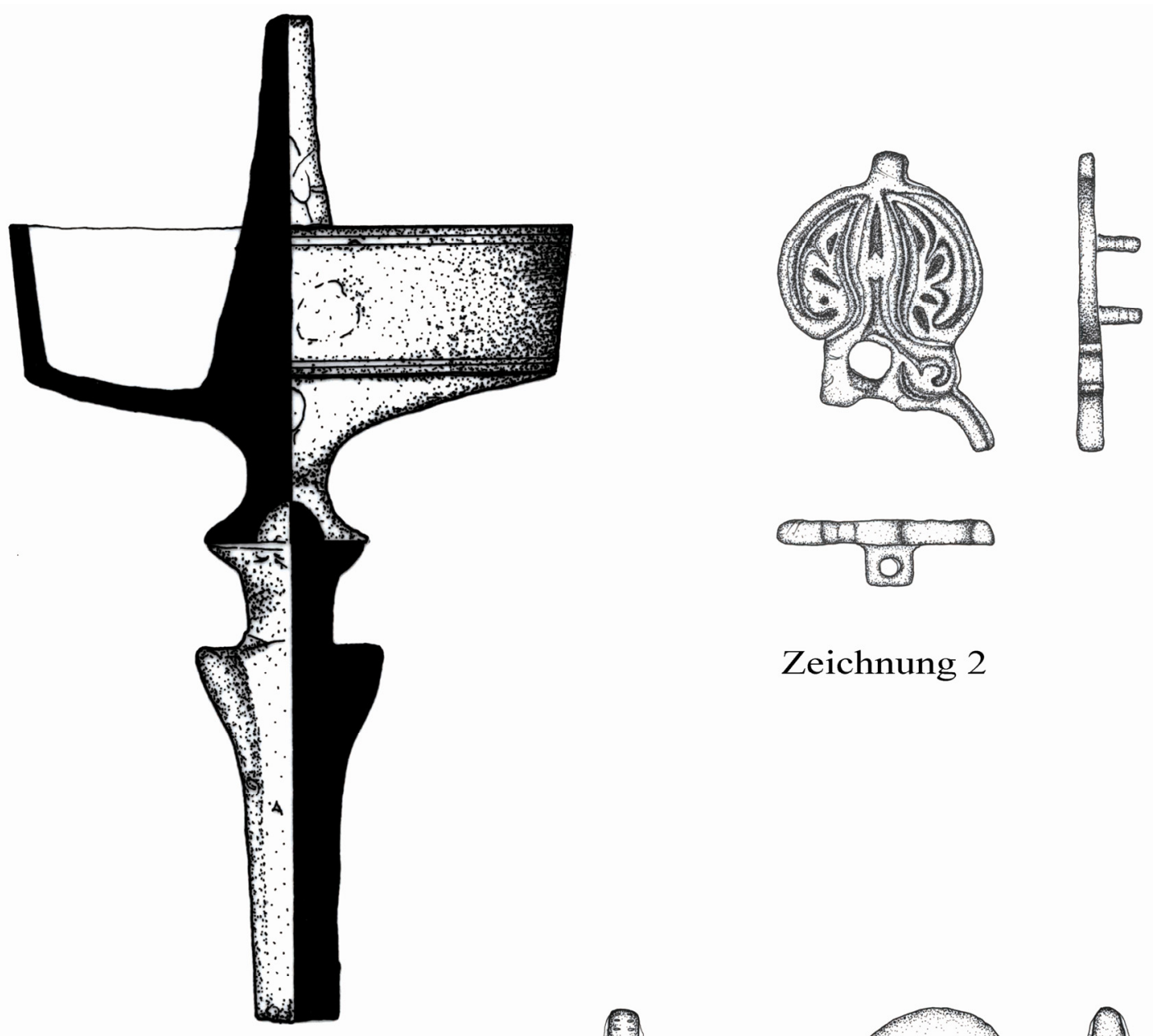

Zeichnung 2

Zeichnung 1
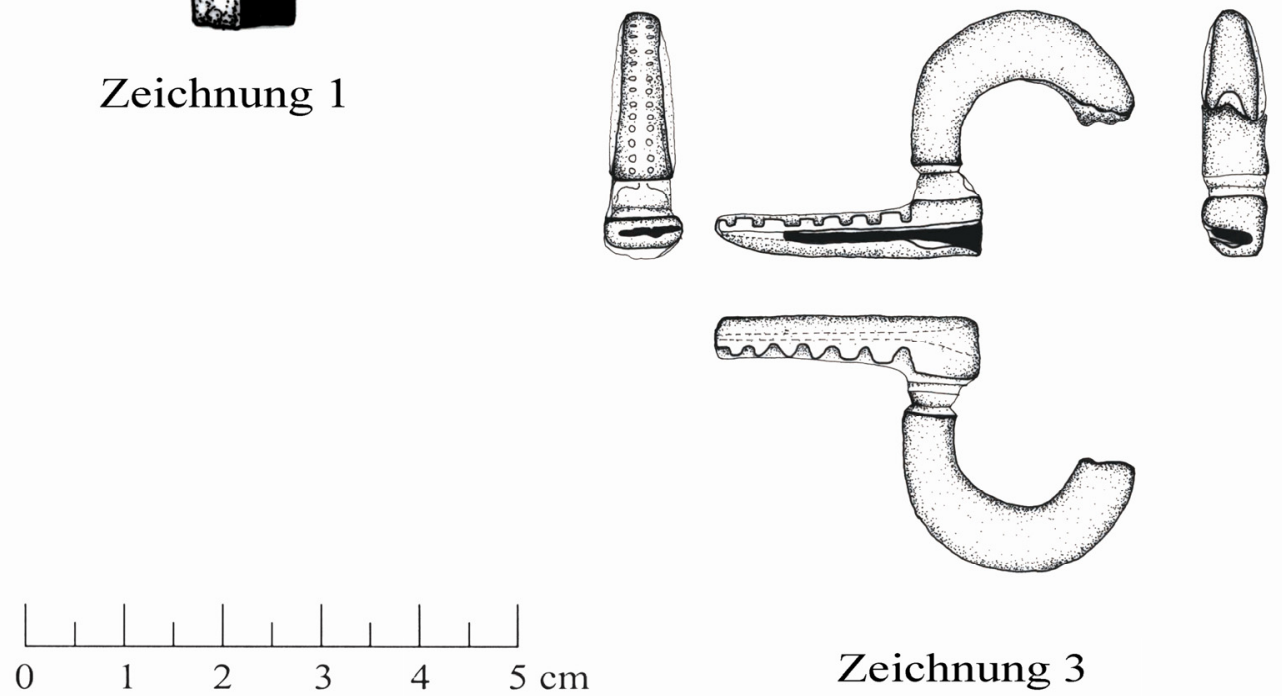

Zeichnung 3 


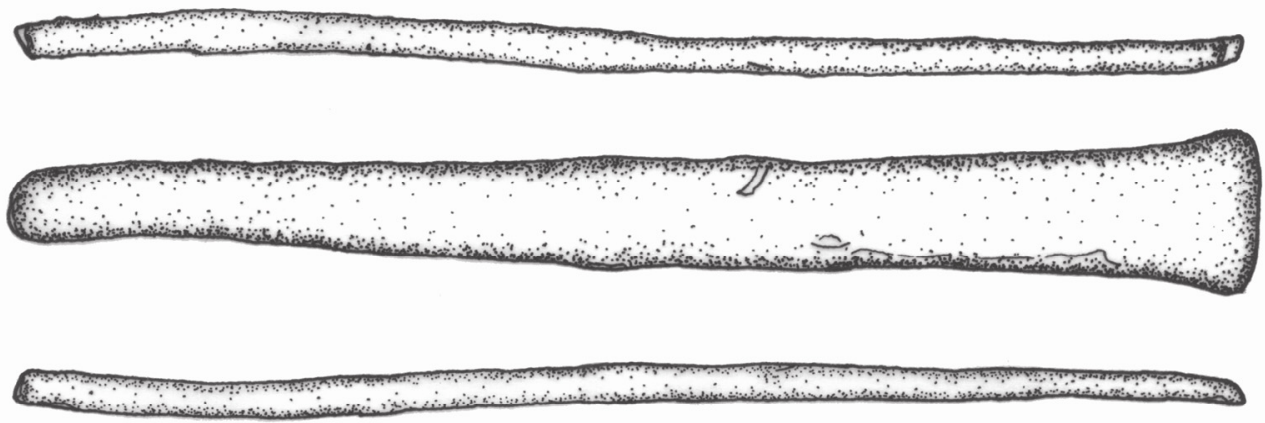

Zeichnung 4

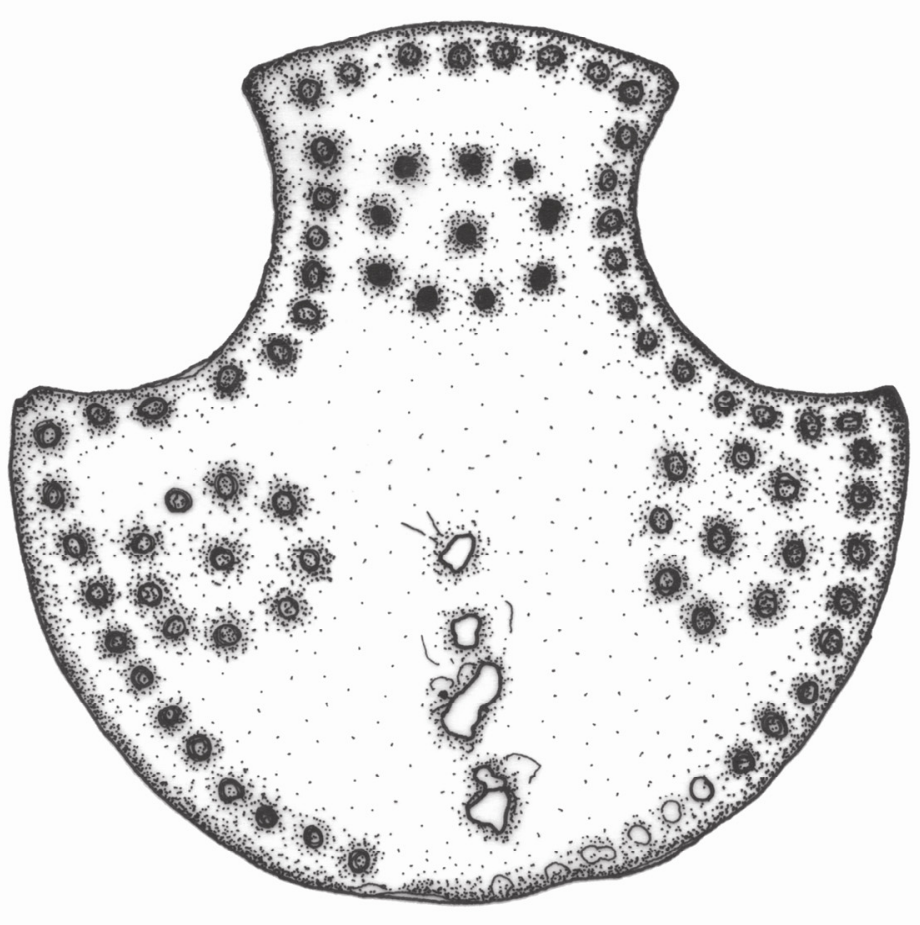

Zeichnung 5

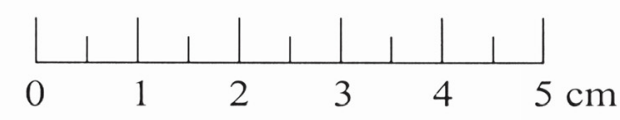



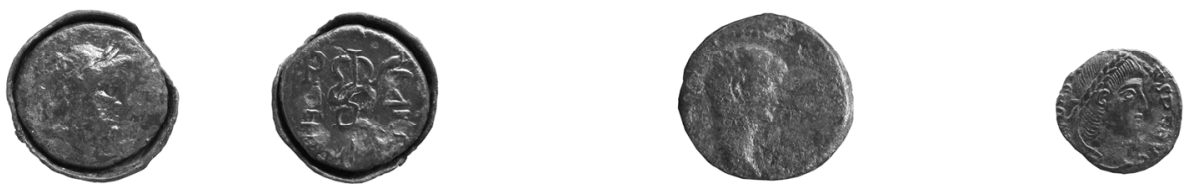

Abb. 1

Abb. 4

Abb. 6
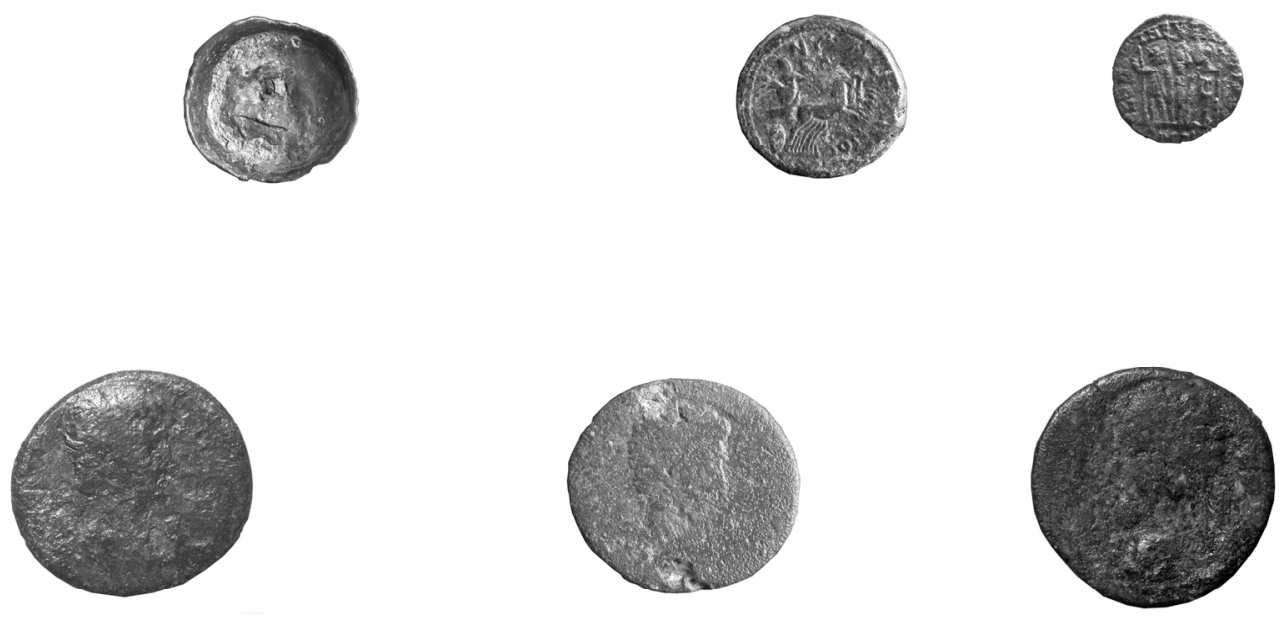

Abb. 2

Abb. 3

Abb. 5
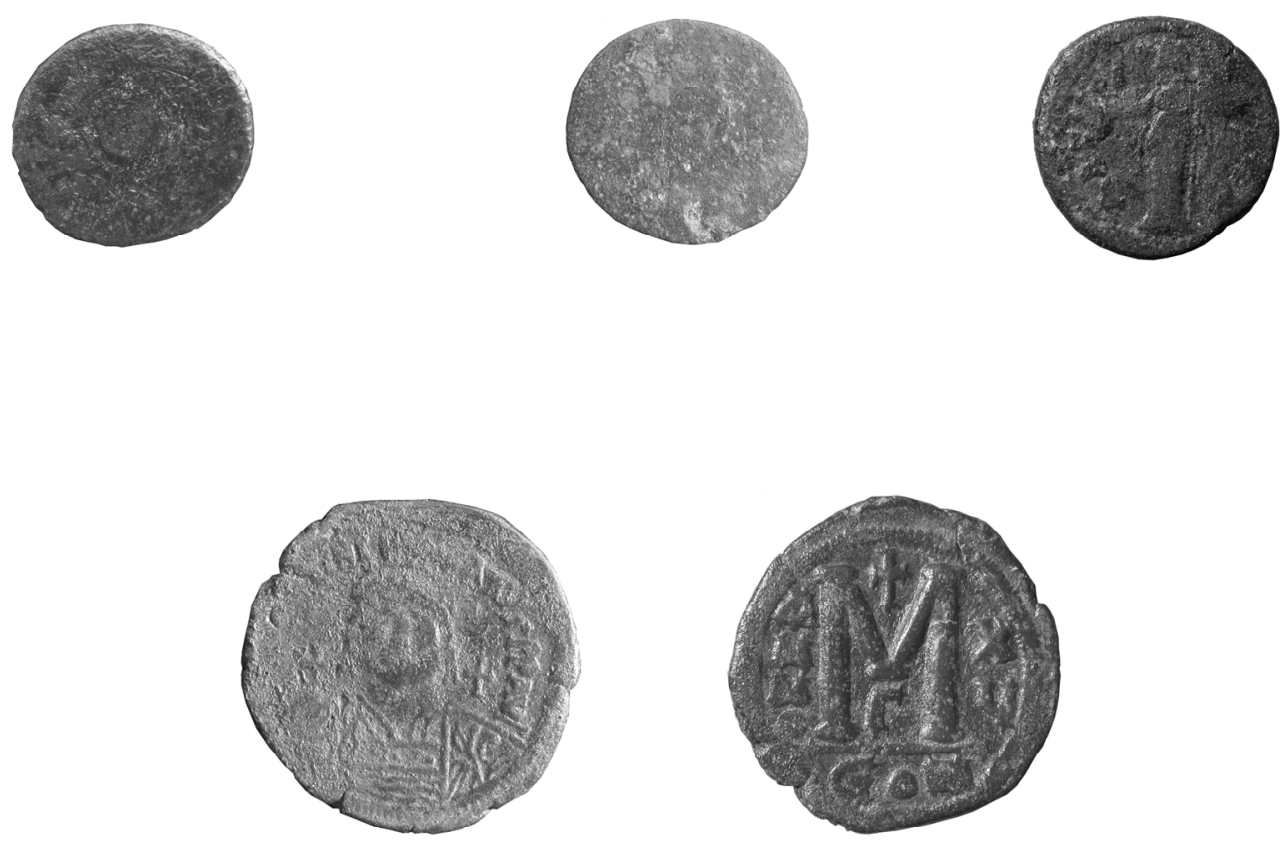

Abb. 7 


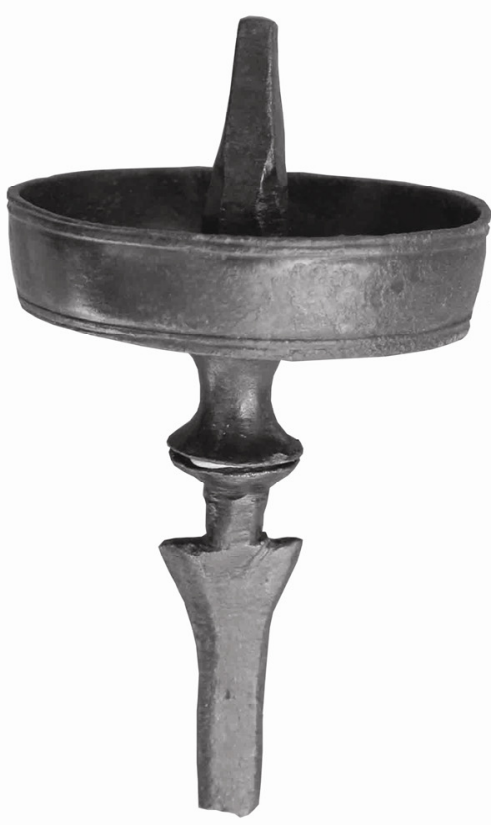

Abb. 8

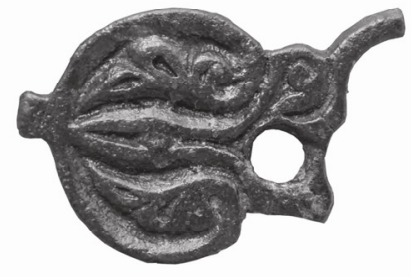

Abb. 9

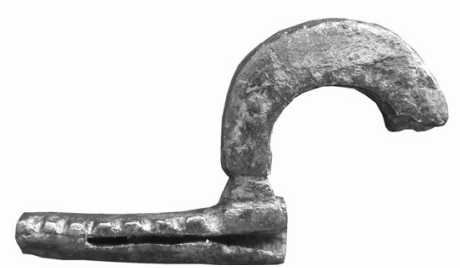

Abb. 10

Abb. 11

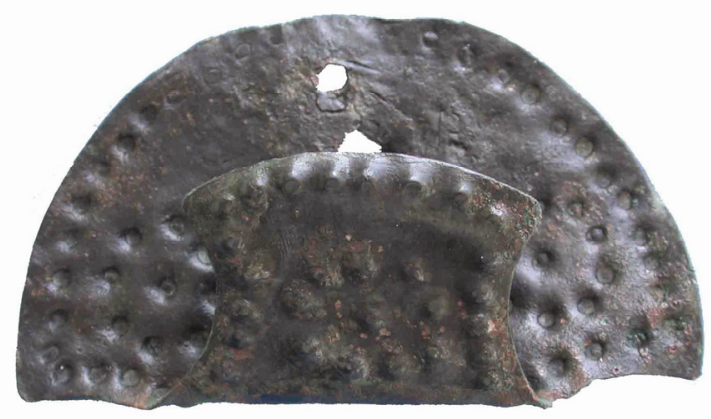

Abb. 12 


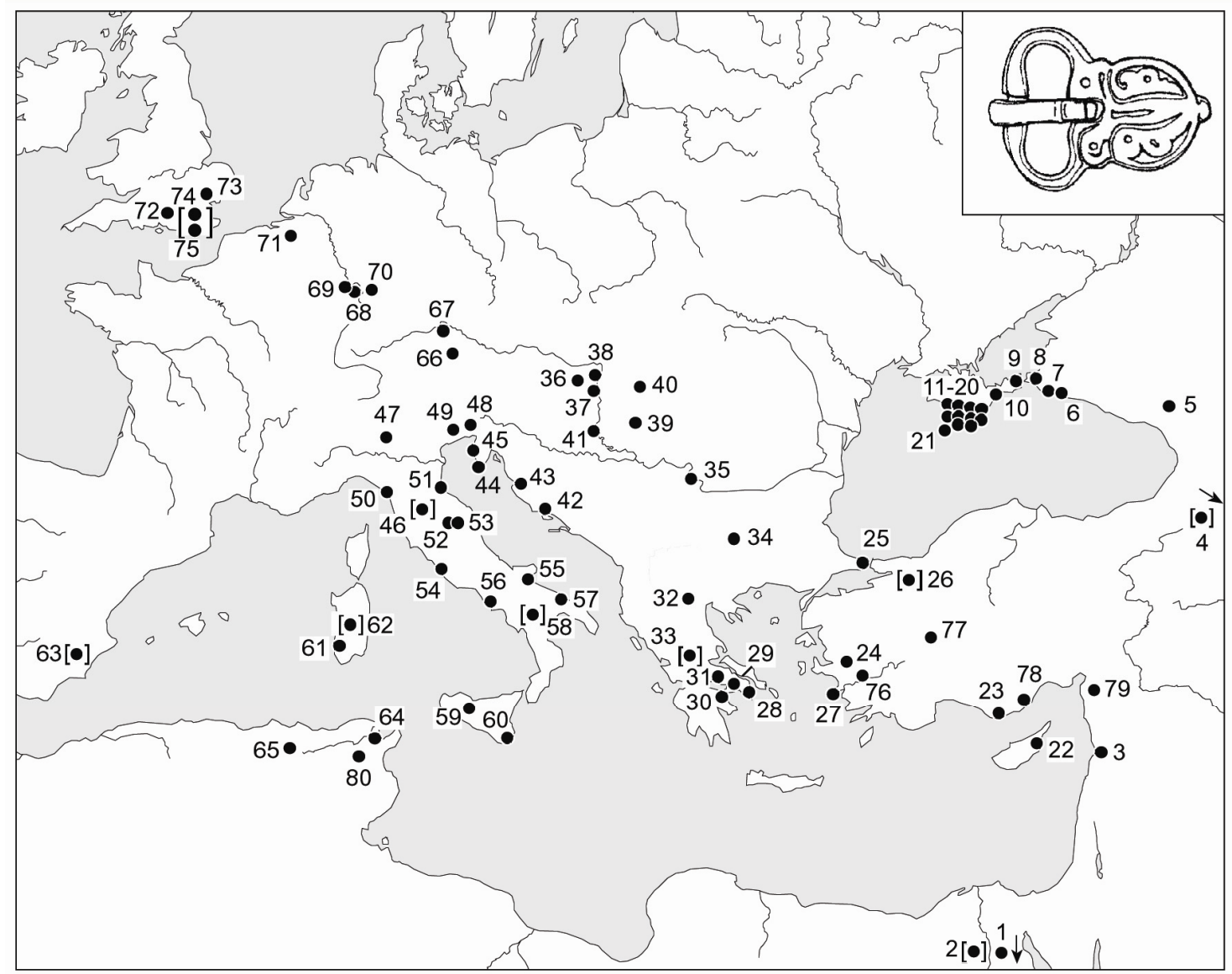

Abb. 13 